\title{
The challenges of chronic pain and fatigue
}

\author{
Authors: Jessica A Eccles ${ }^{A}$ and Kevin A Davies ${ }^{B}$
}

\begin{abstract}
In this review, we explore the challenges of chronic pain and fatigue in clinical practice. Both pain and fatigue are common, troubling and frequently overlapping symptoms, and we describe both the clinical burden and the 'clinical problem'. We explore commonly associated symptoms and possible pathological associations, including variant connective tissue (joint hypermobility), small fibre neuropathy, mast cell activation, dysregulated inflammatory and interoceptive processes, which may inform treatment targets. We suggest a multidisciplinary management approach.
\end{abstract}

KEYWORDS: pain, fatigue, fibromyalgia, hypermobility, rheumatology

DOI: $10.7861 /$ clinmed.2020-1009

\section{Clinical burden of chronic pain and fatigue}

Further understanding of pain and fatigue is clinically important as they are among the most frequent symptoms reported by patients.' When these symptoms are 'persistent' or 'unexplained' they are associated with poorer quality of life and higher costs than other patient groups. ${ }^{2}$ They also pose a diagnostic conundrum and have a significant impact on healthcare utilisation costs and significant indirect costs. ${ }^{3,4}$

Fibromyalgia is characterised by chronic pain, fatigue and what are described as functional symptoms. ${ }^{5}$ Myalgic encephalomyelitis / chronic fatigue syndrome (ME/CFS) is a complex, chronic condition characterised by symptom clusters that include pathological fatigue and malaise that is worse after exertion, cognitive dysfunction, immune dysfunction, unrefreshing sleep, pain, autonomic dysfunction, neuroendocrine and immune symptoms. ${ }^{6}$

Chronic pain affects between one-third and one-half of the population of the UK, whereby fibromyalgia has a reported prevalence of $5 \%{ }^{7}$ In a population-based sample of middle-aged individuals, fatigue was present in one out of five subjects. ${ }^{8}$

Assigning a diagnosis of ME/CFS in the clinical setting often takes years. Many physicians are uninformed or misinformed about the disease. ${ }^{9}$ It has been estimated that $84-91 \%$ of patients affected by ME/CFS are not diagnosed with the

Authors: ${ }^{\mathrm{A}}$ clinical senior lecturer in liaison psychiatry, Brighton and Sussex Medical School, Falmer, UK, Brighton and Sussex University Hospitals NHS Trust, Brighton, UK and Sussex Partnership Foundation NHS Trust, Brighton, UK; ${ }^{\mathrm{B}}$ emeritus professor, Brighton and Sussex Medical School, Falmer, UK and medical director, The Advisory Committee on Clinical Excellence Awards (ACCEA), Leeds, UK condition. ${ }^{10}$ Fibromyalgia and ME/CFS are not uncommon in UK and represent a significant burden to patients and society. Diagnostic delay is significant in both conditions and there are significant difficulties with nosology and nomenclature; presence of shared symptoms has led it to be questioned as to whether fibromyalgia and ME/CFS are manifestations of the same spectrum disorder or separate clinical entities. ${ }^{11-14}$ In our recent study of a mixed group of patients with a clinical diagnosis of fibromyalgia and/or ME/CFS, diagnostic overlap between the two conditions was $\sim 90 \%$. $^{15}$

\section{The real clinical problem}

Physicians of all sorts receive referrals asking for advice on the management of patients with chronic pain and fatigue. Most requests go to rheumatologists, but neurology services are also in the frame, and, in the context of fatigue in particular, advice from colleagues in endocrine, infectious diseases and even cardiology or haematology clinics may be sought, depending often on associated symptoms and comorbidities, clinician preference and availability of appointments. There are huge variations in referral practices across the UK.

So, what happens when a patient is referred to rheumatology, for example? A typical referral letter is illustrated in Box 1. There may be a number of outcomes:

> Patient A's referral passes through a regional triage process of some sort and, for whatever reason, she ends up being seen by a consultant in a busy 'early arthritis' clinic, for example. Royal College of Physicians' guidelines suggest that rheumatology clinics should include 6-7 new patients/clinic, but note that 'in specialised clinics for patients with complex disorders eg early rheumatoid arthritis ... numbers of patients seen in clinics need to be reduced from the recommendations above'. In reality, in the current climate, clinics are far busier, with a mixed population of new and follow-up patients. The focus, as in many branches of medicine, is on protocol-driven assessment, particularly on 'scoring' disease activity, primarily as this dictates the therapeutic options, particularly biologic drug use. How is Patient A likely to fare? She sees Dr A; the clinic is overbooked (22 patients) and she is 'squeezed in' at the end. His clinic letter is illustrated in Box 2.

> Patient $\mathrm{A}$ ends up being seen by $\operatorname{Dr} \mathrm{B}$, a very keen senior research registrar helping out while writing up his $\mathrm{PhD}$ on transgenic murine models of Takayasu's arteritis. He has been primarily working in the lab for the last 2-3 years. His letter is shown in Box 3.

$>$ Having recently abandoned the regional triage process, the department head allocates Patient A to be seen in the newlycreated multiprofessional clinic for complex patients with 
Box 1. A potential referral letter to the rheumatology clinic

Dear colleague,

Could I ask you to see my patient, Patient A? She is 24 -years-old and has just transferred to the practice, having had some issues with her previous general practitioner.

Her main complaints are chronic muscle and joint pain. All muscle groups are involved, but her shoulders and back seem to be the worst areas affected. There is no clear history of joint swelling and, on examination, no deformities or synovitis. She has been troubled by these pains since adolescence, though she informed me she was a keen gymnast at school.

The other concerning issue is severe fatigue. This dates from a holiday in Spain last summer when she apparently developed a febrile illness, precipitating her early return, at which time she also split up from her long-term boyfriend. She now has very poor exercise tolerance, becoming fatigued and dizzy after walking for more than 10 minutes on the flat, and has been off work (she is a university administrative assistant) for the last 3 months, and indicated that she is quite likely to lose her job.

I have accessed her previous records, and I note that she was investigated for an irregular bowel habit while at college, but a faecal calprotectin and other tests were normal, and a putative diagnosis of irritable bowel syndrome was made. This problem seems to have settled. While at school, she was also investigated for a chronic headache, attributed then to excessive screen use. Computed tomography of the brain was normal, and she is no longer under neurological follow-up.

Current medications include etoricoxib, $60 \mathrm{mg}$ once per day, and nortriptyline, $50 \mathrm{mg}$ nocte. She also takes co-codamol, one tablet three times per day, prescribed by a colleague, but does, I think, obtain additional supplies from an online pharmacy, which is clearly a matter of concern. She does not feel that any of these medications really help her pain. I have been reluctant to start gabapentin, or anything similar, in the absence of a clearer diagnosis.

I have performed some baseline blood tests: full blood count, urea and electrolytes, and thyroid function tests are normal; C-reactive protein $<5 \mathrm{mg} / \mathrm{L}$; rheumatoid factor was negative.

Many thanks for your help,

Yours etc

chronic pain and fatigue. She is seen by $\operatorname{Dr} C$, an experienced consultant rheumatologist with a special interest in fibromyalgia and associated conditions and Dr D, a psychiatrist, also with a special interest in this area. Their letters are illustrated in Boxes 4 and 5 .

What should we conclude? Most importantly, what are the likely consequences for the patient of these three different 'approaches'?

The likely outcome in the first scenario and Box 2 is that the patient will seek referral to another specialist, perhaps a

\section{Box 2. Possible response to the potential referral} letter to the rheumatology clinic in Box 1; Dr A

\section{Dear Doctor,}

\section{re Patient A}

Thank you for your helpful letter about Patient $A$. The referral was triaged through the regional referral management service to our EA (early arthritis) clinic and I reviewed her this week.

The history is as you described, with a long history of ill-defined musculoskeletal pain. On examination, there was no evidence of either inflammatory or degenerative arthritis. I note the normal blood count results, low $\mathrm{C}$-reactive protein and negative latex test.

I think we can be confident that this patient does not have a serious musculoskeletal condition. In particular, there is nothing to suggest rheumatoid arthritis, a seronegative arthritis or lupus.

I was able to reassure her that this was the case, and I have discharged her from the clinic.

Yours etc neurologist, endocrinologist or another rheumatologist. She risks the consequences of continued heavy opiate use, inactivity, unemployment and deteriorating mental health.

Should Dr B go to the top of the class, he has, after all, considered lots of the different diagnostic possibilities? Probably not. He makes no reference to having examined the patient, and has not distinguished between 'live' and historical symptoms. Many of his differentials are not credible. The patient has nothing to suggest lupus, for example. His planned programme of investigations (largely unnecessary) will cost several thousands of pounds, he has initiated a protracted long-term follow-up plan, but made no therapeutic recommendations. The patient may still lose her job and she will continue in her 'sick role' as she undergoes more and more investigations.

The third approach (with Boxes 4 and 5) is the appropriate one. The consultation will take no longer than $\mathrm{Dr}$ Bs, not least because of the time saved from not requesting tests. Proper history-taking and examination has enabled the correct diagnosis to be made.

The multiprofessional approach has allowed assessment of the patient's physical and mental health needs, there is a clear management plan going forward, and the patient has been kept fully informed.

So, what is the evidence for an association between chronic pain and fatigue, hypermobility and autonomic dysfunction? What else should we be considering in this type of patient?

\section{Associated symptoms}

In addition to pain and fatigue, a variety of other symptoms are frequently found in fibromyalgia and ME/CFS which have considerable impact on quality of life and functioning. These include over-representation of gastrointestinal, neurological and cardiovascular symptoms, which some have at points been 
Box 3. Possible response to the potential referral letter to the rheumatology clinic in Box 1; Dr B

Dear Doctor,

re Patient $\mathrm{A}$

Thank you for your helpful letter about Patient A. The referral was triaged through the regional referral management service to our EA (early arthritis) clinic and I reviewed her on my consultant's behalf today.

I took the opportunity to review her history in some detail. I note the previous history of gastrointestinal upset and headaches. She was also unwell with a short febrile illness while on holiday last year. As you note, she has widespread muscle pain and marked fatigue, and she also told me that she had had two aphthous mouth ulcers in the last year. Patient A also informed me that she was seen some years ago by Prof $Y$ in London, who suggested the possibility of antinuclear antibody-negative lupus.

I think we need to exclude the possibility that she has inflammatory bowel disease and an associated seronegative arthritis. The headaches also raise the possibility of anti-phospholipid syndrome (a common cause of migrainous headaches in young women), or indeed systemic lupus erythematosus. The latter may, of course, cause myositis, which may be the explanation for her muscle pain.

The recent febrile illness is also a matter of concern, and Lyme disease, acute cytomegalovirus, Epstein-Barr virus or parvovirus are clearly possibilities. Parvovirus infection is, of course, not infrequently complicated by chronic arthritis.

I have therefore arranged for a repeat blood count, urea and electrolytes, and a full auto-antibody profile, including antinuclear antibodies, extractable nuclear antigen, anti-cardiolipin testing, anti-neutrophil cytoplasm antibodies, anti-tissue transglutaminase and Anti-C1q antibodies, as well a full panel of myositis-specific antibodies, including anti-Jo1 antibodies to exclude anti-synthetase syndrome. I am also checking her HLA-B27 status, and also for HLA-B51, to exclude Behçet's disease, in light of the mouth ulceration.

In addition, I have put in train magnetic resonance imaging of her brain and also spinal and sacro-iliac joint magnetic resonance imaging, and requested electromyography and nerve conduction studies.

I have arranged to review her to discuss the blood test results in around 3 weeks, and then again to review the imaging and electrophysiological testing in 3 months' time (there is a considerable wait for these tests at present). If the diagnosis is still unclear at that point, I will consider a positron emission tomography - computed tomography, which is often useful in the setting of undiagnosed inflammatory conditions, such as Takayasu's arteritis.

Yours etc

thought of as functional in origin, and some are increasingly thought to be related to aberrant pain processing (such as central sensitisation or abnormalities of the autonomic nervous system). ${ }^{16,17}$

These include irritable bowel syndrome, where it is reported that there is significant overlap with fibromyalgia and ME/ CFS. ${ }^{18-20}$ Fibromyalgia occurs in up to $60 \%$ of people with irritable bowel syndrome and up to $70 \%$ of people with fibromyalgia have symptoms of irritable bowel syndrome. ${ }^{21}$ Similarly, overlap with irritable bladder or interstitial cystitis is observed. ${ }^{22}$ Likewise, headache is frequently reported in both conditions and is an important source of comorbidity. ${ }^{23}$

There is growing interest in associated dysautonomia, for example, postural orthostatic tachycardia syndrome (PoTS) in which the heart rate rises excessively on standing with attendant symptoms including dizziness, light-headedness, fatigue and palpitations. In addition to orthostatic stress, symptoms can be provoked by heat intolerance and can be worse after eating. ${ }^{24}$ Such abnormalities of the autonomic nervous system are frequently found in both fibromyalgia and ME/CFS. ${ }^{25,26}$

\section{Pathological associations}

\section{Hypermobility}

Joint hypermobility is a common variant of connective tissue affecting up to $20 \%$ of the general population and in symptomatic individuals associated with a wide variety of extraarticular manifestations. ${ }^{27-30}$ Hypermobility is associated with pain, fatigue, gastrointestinal disturbance and headache. ${ }^{31-34}$ It is frequently missed in clinical practice but may provide an important aetiological connection between fibromyalgia, ME/CFS and multiple systemic symptoms, including those described above. ${ }^{35,36}$ Recent work indicates that symptomatic hypermobility may be present in $\sim 80 \%$ of fibromyalgia and ME/CFS patients, yet have only been diagnosed in one out of four cases previously. ${ }^{15}$ Differences in connective tissue matrix (eg collagen) throughout the body could explain disparate symptoms including irritable bowel and bladder, headache and dysfunctional uterine bleeding. Variant connective tissue also provides a putative mechanistic link for brain-body symptoms, whereby differences in connective tissue could lead to impaired vasoconstriction and, subsequently, impaired cardiovascular autonomic function. ${ }^{37}$ Joint hypermobility is firmly associated with dysautonomia with some authors suggesting that at least $50 \%$ of hypermobile patients meet diagnostic criteria for PoTS and up to $80 \%$ all forms of orthostatic intolerance. ${ }^{38-40}$ There is a phenomenological overlap with panic and anxiety, and those with hypermobility are over-represented in panic and anxiety populations by a factor of seven. ${ }^{41}$

\section{Small fibre neuropathy}

Small fibre neuropathy (SFN) is due to structural injury selectively affecting sensory and/or autonomic axons of a small diameter. The clinical presentation is dominated by peripheral neuropathic pain, in particular, but postural hypotension and other symptoms of autonomic dysfunction can occur, and there is clear clinical overlap with the fibromyalgia, in association with anxiety, hypermobility and autonomic dysfunction (FAHA) syndrome which we describe earlier. ${ }^{42}$ Autonomic symptoms may predominate 
Box 4. Possible response to the potential referral letter to the rheumatology clinic in Box 1; Dr C

Dear Doctor,

re Patient $\mathrm{A}$

Thank you for your helpful letter about Patient A. I saw her in my multidisciplinary rheumatology new patient clinic this week.

Reviewing her history, the active clinical problems today are widespread muscle pain and sensitivity, extreme fatigue and poor exercise tolerance, limited mainly by lack of energy, but also by a feeling of dizziness. This last symptom has bothered her for many years, and she has had a number of 'faints', one of which resulted, 2 years ago, in a visit to the emergency department. At which point, she had electrocardiography which showed a sinus tachycardia, but no other abnormality.

The illness you mention is Spain sounds very much like an attack of holiday diarrhoea. She still experiences occasional intestinal hurry, but this is usually associated with the use of over-the-counter proton pump inhibitors, which she uses regularly on an as needed basis.

Since she was investigated in her teens, she has had only occasional headaches, but she does complain of poor concentration and 'brain fog'.

She sleeps poorly, and wakes feeling unrefreshed. Due to her fatigue, she often sleeps for short periods in the day.

Patient A also describes having a dry mouth, much worse since starting treatment with nortriptyline, but no ocular sicca, and only a very occasional mouth ulcer. Her weight is stable, and appetite fair. As you note, she has been off work for some time, and she was informed that an occupational health review is pending, something about which she is understandably very concerned. She does not have a regular partner at present and is not sexually active.

On examination, Patient A looked well, but came across as very anxious. She was able to give a very good account of herself and brought along copies of a number of letters from specialists she had seen in the past. There was no lymphadenopathy, clubbing or rash, and a Shirmer's test was normal. Her resting pulse rate was 98 beats per minute, blood pressure (prone) 130/90 mmHg; blood pressure (standing) 100/70 mmHg. Cardiovascular, respiratory and abdominal examinations were all otherwise normal. Reflexes were brisk and symmetrical, with no evidence of a peripheral neuropathy or radiculopathy and, with encouragement, normal power in all muscle groups. Musculoskeletal examination revealed no joint deformity or swelling, a good range of back movement and no evidence of sacroiliac pathology. However, Patient A is clearly a hypermobile individual: Beighton score $=9$, with markedly hyperextensible knees and elbow joints in particular. There was localised pain and tenderness referable to the parasternal area bilaterally, both elbows, buttocks and the muscles of the shoulders and neck on both sides.

My view is that this patient has fibromyalgia, in association with anxiety, hypermobility and autonomic dysfunction (manifest as postural orthostatic tachycardia syndrome (PoTS)), ie FAHA. Many features in the history and examination support this diagnosis, notably the non-restorative sleep pattern and fatigue, widespread pain, and previous history of irritable bowel syndrome, 'brain fog' and headaches.

I am rechecking her blood count and thyroid function tests, as well as calcium, magnesium (low magnesium is common in proton pump inhibitor (PPI) users) and vitamin D levels. No other blood tests or imaging are required. I shall discuss the results with her as part of a planned telephone follow up in 3 weeks.

My colleague Dr D also reviewed the patient in our multidisciplinary clinic, and she was also assessed by our speciality physiotherapist, who is planning a graded exercise programme, and has referred her to our nurse-led fibromyalgia support group for advice on painmanagement in particular. Dr D's letter is appended.

I explained the clinical problem to Patient A, particularly the relevance of her hypermobility, and provided her with the Versus Arthritis information leaflets on both hypermobility and fibromyalgia, as well as a number of links to informative websites, with a view to discussing any specific issues at our future telephone consultation.

We agreed to cease her PPI use, aim to reduce and stop her opiates, and also her etoricoxib in due course. I would recommend continuing nortriptyline for the time being and you may also wish to consider melatonin, $2 \mathrm{mg}$ nocte for 2 months or so to try to normalise her sleep pattern. I have also agreed, with appropriate consent, to provide a report for the occupational health department at the university.

Yours etc

in some patients in particular. The more 'typical' picture is of a 'length-dependent' neuropathy, with symptoms starting in the feet and progressing proximally, eventually resulting in a "glove and stocking' picture. As in fibromyalgia, the pain may be chronic, but there may be a marked stimulus-dependent component (eg a sufferer may not be able to tolerate wearing tights or socks, or complain of pain associated with the weight of a duvet). In related conditions, increased temperature may cause 'attacks', but this type of presentation is more common in certain rare 'channelopathies' (eg erythromelalgia and paroxysmal extreme pain disorder (PEPD)).

SFN may be associated with diabetes, HIV (and certain reverse transcriptase inhibitors), alcohol use, Sjogren's syndrome and lupus, among others. Amyloid polyneuropathy can present as SFN However, at least $50 \%$ of cases are idiopathic but, of this group, around one-third have variants of the SCN9A gene, encoding the Nav1.7 sodium channel, a voltage-gated ion channel that is selectively expressed in sensory and autonomic neurones. ${ }^{43}$ These 
Box 5. Possible response to the potential referral letter to the rheumatology clinic in Box 1; Dr D

Dear Doctor,

re Patient $\mathrm{A}$

I reviewed Patient $A$ in our multidisciplinary clinic from a liaison psychiatry perspective. As $\operatorname{Dr} C$ suggests, there is a complex interplay between her physical symptoms which are likely unified by hypermobility. She reports, as is often found in similar presentations, a degree of anxiety with panic symptoms and some issues relating to her change in circumstances and impact of physical symptoms on mental health.

She is going to engage with our physiotherapist to improve strength and stability with the hope of reducing pain and fatigue. Given her psychological symptoms and the symptoms suggestive of orthostatic intolerance (again very common in hypermobile presentations), I would suggest a trial of a low-dose beta blocker (providing no contraindications) to manage both anxiety and dysautonomic symptoms.

She will likely benefit from a biopsychosocial approach, so I am referring her to our health psychologist and also signposting Patient A for vocational support and advice regarding reasonable adjustments and additional support to help her remain in work.

Yours etc

Nav1.7 variants cause increased excitability of sensory neurones and eventual degeneration of small fibres. SFN may also be associated with variants in the SCN1OA gene (encoding the Nav1.8 channel), which enhance the excitability of dorsal root ganglion cells.

There are two main reasons that the real incidence is unknown. Firstly, there are no internationally agreed diagnostic criteria for SFN and, more importantly, the diagnosis is not easy to make. Bedside clinical neurological examination is usually normal, including tendon reflexes, coordination and large fibre sensory function (such as light touch, vibration sensation and proprioception). There may be a distal loss of pinprick or thermal sensation. In reality, accurate testing of thermal sensitivity is rarely performed and, in inexpert hands, unlikely to be reliable. Conventional electrophysiological testing is also normal. The gold standard for diagnosis is quantification of intra-epidermal nerve fibre density in a skin biopsy (usually a punch biopsy from a distal site) by histochemical staining for an antigen called PGP 9.5 (an ubiquitin hydrolase) that is found in all nerve fibres. This is a highly specialised technique, only performed in specialist centres.

In summary, it is certainly possible that significant numbers of patients with clinical presentations similar to Patient A (illustrated in Box 1) may have SFN. The presence of a truly a 'lengthdependent' neuropathy, should alert a physician to this possibility, but the variation in the clinical presentation of the condition, and the difficulty in establishing a firm diagnosis, mean that many patients are, in all probability, currently 'missed'.

\section{Mast cell activation}

There is increasing interest in the role of mast cells (MCs) in chronic disease. There are several patient groups in the UK, USA and elsewhere that actively espouse the importance of 'mast cell activation disorders' (MCAD) in patients with chronic fatigue, and dysautonomia, particularly in the context of hypermobility (hypermobile type Ehlers-Danlos syndrome (hEDS)). Is this plausible? If so, what, if any, is the clinical relevance?

Mature differentiated MCs are found only within tissues, where they congregate around nerves, blood vessels and lymphatics. Two main types of MCs have been described. Found in connective tissue, skin and the peritoneal cavity) they contain tryptase (MCT) in their granules and express interleukin-5 (IL-5) and IL-6. MCs which are home to the respiratory mucosa and gut contain tryptase and chymase (MCTC), and express IL- $4 .{ }^{44}$ Activated, mast cells produce a range of pre-formed and newly synthesised mediators. Within a few minutes of activation, pre-formed mediators (histamine and proteases) are released, following which, there is de novo synthesis of membrane-derived lipid mediators (prostaglandins and leukotrienes) and a range of pro- and antiinflammatory cytokines and chemokines.

In the context of disease, MCs are typically recognised for their role in immediate immunoglobulin (Ig) E-mediated, allergic responses in anaphylaxis, physical urticarias, angioedema, food allergy, venom allergy and asthma. However, pathological MC activation has also been implicated in nonallergic disorders, including headache syndromes, irritable bowel syndrome, non-celiac gluten enteropathy, autoimmune syndromes and neuropsychiatric disorders, in addition to rare 'primary' mast cell disorders such as systemic mastocytosis (SM) and monoclonal mast cell activation syndrome. There are agreed diagnostic criteria for SM but not for other types of MCAD. ${ }^{45}$

Symptoms of MCAD include urticaria, pruritus, flushing, intestinal hurry, abdominal cramping, osteoporosis, headache, postural hypotension and neuropsychiatric symptoms. Particularly relevant to this review are the recently described associations with hypermobility and hEDS. ${ }^{46}$

Immunohistochemical analysis identified an increased content of chymase-positive MCs in the undamaged skin of patients with signs suggestive of 'true' connective tissue disorders. ${ }^{47}$ Other investigators have described symptoms compatible with a nonIgE-mediated MCAD in patients with joint hypermobility. The most common symptoms reported are naso-ocular symptoms, and a history of anaphylaxis or asthma. As long ago as 2015, a possible new syndrome was suggested involving mast cell activation syndrome (MCAS), PoTS and hypermobility (EDS). In one early cohort study, screening of patients with PoTS and EDS revealed that two-thirds reported symptoms consistent with MCAS. The association has recently been reviewed in depth by Kohn and Chang. ${ }^{48}$

So, how is MCAS diagnosed and managed? Testing relies on the measurement of MC mediators. It is suggested that ideally, serum tryptase and chromogranin A, plasma histamine, prostaglandin (PG) D2 and heparin, as well as urinary (random and 24-hour) histamine, $\mathrm{N}$-methylhistamine (NMH), 11- $\beta$-PGF2 $\alpha$, and leukotriene (LT) E4 should be assessed. These tests are not widely available, but some can be organised through specialist centres. If MCAS is suspected in the context of hypermobility, PoTS and/or other suggestive symptoms, one pragmatic approach is to initiate a trial of therapy with a combination of $\mathrm{H} 1$ and $\mathrm{H} 2$ blockers, in combination with cromoglycate, or a leukotriene receptor blocker. Like SFN, discussed earlier, physicians are limited by the lack of readily available tests, clear diagnostic criteria or treatment protocols. However, it seems likely that mast cell disorders will be 
increasingly recognised in the future and MCAS will become a more 'mainstream' diagnosis.

\section{Inflammatory reactivity}

There is evidence of raised inflammatory markers in fibromyalgia and ME/CFS and proteomic analysis suggests that upregulated inflammation can play a major role in the aetiology of fibromyalgia. ${ }^{49,50}$ Preliminary results from our recent study into autonomic and inflammatory mechanisms of pain and fatigue suggest correlation between baseline inflammatory markers and pain and fatigue symptoms. ${ }^{51}$

However, in ME/CFS conventional markers of inflammation (eg (RP and ESR) are normal. Non-steroidal anti-inflammatories are rarely of long-term benefit, and the associated gastrointestinal side effects and enhanced cardiovascular risks mitigate against the use of these drugs. Corticosteroids are also not indicated, and may be positively harmful, resulting in altered mood, osteoporosis, impaired glucose tolerance and hypertension, as well as 'steroid dependency' as a consequence of adrenal suppression.

\section{Interoceptive processing}

Differences in interoceptive processing (relating to the internal sensation of what is happening in the body) maybe key in delineating the underlying brain-body mechanisms in such conditions, including hypermobility. ${ }^{52,53}$ Recent work suggests fatigue may be related to disrupted interoceptive mechanisms, equally, pain is also linked to differences in interoception. ${ }^{54-57}$ Mismatch between such observed and expected sensory signals representing bodily states may be important in symptom generation and maintenance, and such models have been extended to fatigue. ${ }^{58,59}$ Thus interoceptive mismatch may be a factor in generation and maintenance of symptoms in such conditions, and may provide possibilities for treatment targets (eg https://doi.org/10.1186/ISRCTN17018615). ${ }^{60}$

\section{What about long COVID?}

The UK and much of the rest of the world are still battling the consequences of the 'second wave' of SARS-CoV-2 infection. In the UK, the severity of this is perhaps greater than anticipated, primarily due to lack of a general desire to support effective public health measures in a timely fashion, sociopolitical and commercial imperatives prevailing over high-quality scientific advice. However, while clinicians' capacity to manage the acute illness associated with infection has improved greatly over the last 9 months, mortality is still high in vulnerable groups. There is currently an increasing focus on the longer-term consequences of infection. Chronic illness, particularly affecting the respiratory system, after infection was well-described in the original SARS outbreak many years ago affecting up to $30 \%$ of patients, and Carfi and colleagues first identified a similar problem with SARSCoV-2 infection in a large group of Italian patients, with a reported persistence of at least one symptom, particularly fatigue and dyspnoea, in nearly $90 \%$ patients who had recovered from acute infection. ${ }^{61,62}$ This is now widely recognised and was the subject of an authoritative review published recently by the National Institute for Health Research (NIHR). ${ }^{63}$ Common symptoms include breathlessness, cough and palpitations, but also chronic fatigue (which can be severe), myalgia and joint pain, anxiety, low mood, cognitive impairment ('brain fog') and atypical chest pain. In the NIHR review, the authors conclude:

Indeed there is a possibility that the symptoms described may be due to a number of different syndromes (eg post-intensive care syndrome, post-viral fatigue syndrome and long-term COVID syndrome). Some people may be suffering with more than one syndrome at the same time.

A proportion of patients presenting with ill-health post-COVID will be found to have evidence of residual 'physical' damage affecting the lungs or cardiovascular system. This does not appear to be the case in the majority of patients, however, nor is there any objective evidence of an on-going 'inflammatory' process nor the development of autoimmunity or a conventional 'reactive' illness. The non-cardiopulmonary symptoms described map closely onto those which constitute the subject of this review. This raises the possibility that long COVID should, in the absence of proven residual tissue injury, be regarded as part of the CFS spectrum, and managed as such. Acute SARS-CoV-2 infection is an exogenous 'stressor', which has precipitated the condition. Individual physiological and psychological responses to such stressors are highly variable, as discussed earlier. Concomitant psychosocial stress factors, related to economic uncertainties, employment issues, and disruption of normal social and familial structures and processes, both at the level of the individual and within a wider societal context, mean that it may be very difficult to develop the appropriate coping strategies that allow full recovery from acute illness. We should not, therefore, be surprised that the condition that has been labelled as long COVID appears to be increasingly common. It is also true that, in acute SARS-CoV-2 infection, there is, of course, a need to make a specific 'virological' diagnosis to inform management and to aid efforts to reduce the spread of the virus in the population. The condition is now relatively 'common' and still increasing in prevalence. No such specific virological diagnosis is usually made in the majority of patients with "postviral' syndromes.

Long COVID is, at the time of writing, still very poorly understood, however, and there are a number of current centrally-funded research initiatives designed to address (among other things) whether there is objective evidence of chronic nervous system damage (central, peripheral or autonomic) and whether there are specific genetic factors that may pre-dispose an individual to chronic illness after acute infection.

We discuss later the importance of identifying other conditions that may present with chronic fatigue, pain or related symptoms, and this is, of course, no less important in the context of long COVID. Throughout this article we have, however, emphasised the need for a holistic, multi-professional approach to our patients. It is encouraging to hear that the NHS is investing in 40 centres precisely for this purpose. We hope, however, that these centres will not restrict their remit exclusively to the care of long COVID sufferers. Encouraging news on vaccine development may mean that COVID-19 is 'defeated' and long COVID may hopefully be of little clinical importance in 5 years' time. Patients, such as our index case (Patient A), will, on the other hand, always be there.

\section{Am I missing something?}

Outpatient assessment of a patient with fatigue and chronic pain can be challenging. This is particularly the case when the patient is polysymptomatic and they have had many interactions with 
Table 1. Assessment of conditions that are frequently missed and easily identified

\begin{tabular}{|c|c|c|c|}
\hline $\begin{array}{l}\text { Clinical feature or } \\
\text { abnormal test }\end{array}$ & Other features & Corroborative tests & Differential diagnoses \\
\hline Serostis & Documented fever or arthropathy & CRP, FBC or genetic testing & Familial Mediterranean fever \\
\hline $\begin{array}{l}\text { Mucosal ulcers (oral/ } \\
\text { genital) }\end{array}$ & Arthropathy, pathergy or rash/EN & HLA-B51 & Behçet's disease \\
\hline Sicca: oral/ocular & Arthropathy or chronic rash & Shirmer's test or auto-antibodies & Sjogren's syndrome \\
\hline Osmotic symptoms & Weight loss or abdominal pain & Glucose, $\mathrm{HbA} 1 \mathrm{c}$ or calcium & Diabetes or hypercalcaemia \\
\hline Arthropathy & $\begin{array}{l}\text { Photosensitivity, Raynaud's } \\
\text { syndrome or rash }\end{array}$ & ANA, ENA, complement or ferritin & $\begin{array}{l}\text { Lupus, adult Still's disease or } \\
\text { Lyme disease }\end{array}$ \\
\hline Urticaria & $>48$ hours or residual discolouration & ANA, anti-C1q Ab or complement & Urticarial vasculitis (HUVS) \\
\hline Abdominal/pelvic pain & $\begin{array}{l}\text { Dyspareunia, menorrhagia or } \\
\text { infertility }\end{array}$ & Pelvic imaging or laparoscopy & Endometriosis or Crohn's disease \\
\hline $\begin{array}{l}\text { Extreme daytime } \\
\text { somnolence }\end{array}$ & Snoring, obesity or airway issues & Specialist sleep study & Obstructive sleep apnoea \\
\hline Lymphopenia & EN, lupus symptoms or infections & SACE, ANA or HIV testing & Sarcoid, lupus or HIV \\
\hline
\end{tabular}

healthcare professionals before. It is all too easy to 'admit defeat' as in Dr A's case, or initiate a test or another referral in 'response' to every symptom described by the patient during the course of their illness (Dr B). How can this be avoided?

One approach is to adopt the 'snapshot' approach and ask the patient to identify a maximum of three 'live' clinical problems (eg in the previous 2 weeks), highlighting which is the most significant one. If the primary problem is fatigue or pain (as in our exemplar Patient A), it can be highly informative to identify the single most important additional symptom: the 'plus one' approach. This should raise a specific subset of diagnostic possibilities, often limited in number, which will prompt further, more focused 'direct' questioning, and may inform an initial investigation strategy, if considered appropriate.

All physicians are trained to identify 'red flag' symptoms, such as unexplained weight loss, which, in older patients particularly, may point to a diagnosis of malignancy, malabsorption, endocrine disease or depression. However, there is a number of conditions that are frequently missed, and easily identified. Some of these are summarised in Table 1. We focus on the 'plus one'. The average time it takes to diagnose Behçet's, for example, may be up to 7 years and there is frequently a delay in the diagnosis of Sjogren's syndrome; few doctors ask about sicca. ${ }^{64}$ Inflammatory bowel disease or endometriosis can also easily be missed, as can HIV, especially in older patients. ${ }^{65,66}$.

What is rarely helpful or informative in a busy clinic setting is to rehearse in detail with a patient the detailed chronology of what may be several years of ill-health. This can all too often result in a 'memory test' for the patient, with the physician seeking to corroborate dates and details in the notes or electronic patient record. This wastes time, and can be frustrating for all concerned. However, it may be useful before, or more often after, the consultation to look back at previous letters, imaging or pathology results to help substantiate or refute a presumptive diagnosis. In patients with a 'long history' and multiple medical interactions, it is, however, important to avoid 'medical transference' and give undue credence to diagnoses made by other doctors, often years before. Firstly, the patient's recollection of the outcome of a previous consultation may be far from accurate, and secondly, the professor may well have been wrong. It does happen.

\section{The future}

In this short review we have highlighted what we feel to be a real association between chronic pain and fatigue, hypermobility, anxiety and dysautonomia, and suggested how these things may be linked pathophysiologically. We feel that the explicit recognition of this phenotype is of potentially of great clinical utility, particularly in planning care at the level of the individual patient. In addition, we would strongly espouse the development of effective multiprofessional clinical services as are planned, for example, for long COVID sufferers, to serve the true clinical needs of such patients, addressing both physical and mental health needs, avoiding unnecessary investigations, and maximising the opportunity to make a clinical diagnosis of other associated conditions.

\section{References}

1 Finley CR, Chan DS, Garrison S et al. What are the most common conditions in primary care? Systematic review. Can Fam Physician 2018;64:832-40.

2 Zonneveld LNL, Sprangers MAG, Kooiman CG et al. Patients with unexplained physical symptoms have poorer quality of life and higher costs than other patient groups: a cross-sectional study on burden. BMC Health Services Research 2013:13:520.

3 Winkelmann A, Perrot S, Schaefer C et al. Impact of fibromyalgia severity on health economic costs: results from a European crosssectional study. Appl Health Econ Health Policy 2011;9:125-36.

4 Collin SM, Bakken IJ, Nazareth I et al. Health care resource use by patients before and after a diagnosis of chronic fatigue syndrome (CFS/ME): a clinical practice research datalink study. BMC Family Practice 2017;18:60. 
5 Atzeni F, Talotta R, Masala IF et al. One year in review 2019: fibromyalgia. Clin Exp Rheumatol 2019;37 Suppl 116:3-10.

6 Bested AC, Marshall LM. Review of Myalgic Encephalomyelitis/ Chronic Fatigue Syndrome: an evidence-based approach to diagnosis and management by clinicians. Rev Environ Health 2015;30: 223-49.

7 Fayaz A, Croft P, Langford RM et al. Prevalence of chronic pain in the UK: a systematic review and meta-analysis of population studies. BMJ Open 2016;6:e010364.

8 Galland-Decker C, Marques-Vidal P, Vollenweider P. Prevalence and factors associated with fatigue in the Lausanne middle-aged population: a population-based, cross-sectional survey. BMJ Open 2019;9:e027070.

9 Clayton EW. Beyond myalgic encephalomyelitis/chronic fatigue syndrome: an IOM report on redefining an illness. JAMA 2015;313: 1101-2.

10 Solomon L, Reeves WC. Factors influencing the diagnosis of chronic fatigue syndrome. Arch Intern Med 2004;164:2241-5.

11 Choy E, Perrot S, Leon T et al. A patient survey of the impact of fibromyalgia and the journey to diagnosis. BMC Health Serv Res 2010;10:102.

12 Comiskey C, Larkan F. A national cross-sectional survey of diagnosed sufferers of myalgic encephalomyelitis/chronic fatigue syndrome: pathways to diagnosis, changes in quality of life and service priorities. Ir ] Med Sci 2010;179:501-5.

13 Hyland ME, Bacon AM, Lanario JW et al. Symptom frequency and development of a generic functional disorder symptom scale suitable for use in studies of patients with irritable bowel syndrome, fibromyalgia syndrome or chronic fatigue syndrome. Chronic Dis Transl Med 2019;5:129-38.

14 Natelson BH. Myalgic encephalomyelitis/chronic fatigue syndrome and fibromyalgia: definitions, similarities, and differences. Clin Ther 2019;41:612-8.

15 Eccles JA, Thompson B, Themelis K et al. Beyond bones: The relevance of variants of connective tissue (hypermobility) to fibromyalgia, ME/CFS and controversies surrounding diagnostic classification: an observational study. Clin Med 2021;21:53-8.

16 Arendt-Nielsen L, Morlion B, Perrot S et al. Assessment and manifestation of central sensitisation across different chronic pain conditions. Eur J Pain 2018;22:216-41.

17 Mathias CJ, Low DA, Iodice $V$ et al. Postural tachycardia syndromecurrent experience and concepts. Nat Rev Neurol 2011;8:22-34.

18 Yang TY, Chen CS, Lin CL et al. Risk for Irritable Bowel Syndrome in Fibromyalgia Patients: A National Database Study. Medicine (Baltimore) 2017;96:e6657.

19 Martínez-Martínez LA, Mora T, Vargas A et al. Sympathetic nervous system dysfunction in fibromyalgia, chronic fatigue syndrome, irritable bowel syndrome, and interstitial cystitis: a review of casecontrol studies. J Clin Rheumatol 2014;20:146-50.

20 Petersen MW, Schröder A, Jørgensen T et al. Irritable bowel, chronic widespread pain, chronic fatigue and related syndromes are prevalent and highly overlapping in the general population: DanFunD. Scientific Reports 2020;10:3273.

21 Wallace DJ, Hallegua DS. Fibromyalgia: The gastrointestinal link. Curr Pain Headache Rep 2004:8:364-8.

22 Nickel JC, Tripp DA, Pontari M et al. Interstitial cystitis/painful bladder syndrome and associated medical conditions with an emphasis on irritable bowel syndrome, fibromyalgia and chronic fatigue syndrome. J Urol 2010;184:1358-63.

23 Ravindran MK, Zheng Y, Timbol C et al. Migraine headaches in chronic fatigue syndrome (CFS): comparison of two prospective cross-sectional studies. BMC Neurol 2011;11:30.

24 Arnold AC, Ng J, Raj SR. Postural tachycardia syndrome - Diagnosis, physiology, and prognosis. Auton Neurosci 2018;215:3-11.

25 Reynolds GK, Lewis DP, Richardson AM et al. Comorbidity of postural orthostatic tachycardia syndrome and chronic fatigue syndrome in an Australian cohort. J Intern Med 2014;275:409-17.
26 Cutsforth-Gregory JK, Sandroni P. Clinical neurophysiology of postural tachycardia syndrome. Handb Clin Neurol 2019;161:429-45.

27 Malfait F, Castori M, Francomano CA et al. The Ehlers-Danlos syndromes. Nat Rev Dis Primers 2020;6:64.

28 Mulvey MR, Macfarlane GJ, Beasley M et al. Modest association of joint hypermobility with disabling and limiting musculoskeletal pain: results from a large-scale general population-based survey. Arthritis Care Res (Hoboken) 2013;65:1325-33.

29 Castori M. Ehlers-Danlos syndrome, hypermobility type: an underdiagnosed hereditary connective tissue disorder with mucocutaneous, articular, and systemic manifestations. ISRN Dermatol 2012;2012:751768.

30 Tinkle BT, Levy HP. Symptomatic joint hypermobility: the hypermobile type of Ehlers-Danlos syndrome and the hypermobility spectrum disorders. Med Clin North Am 2019;103:1021-33.

31 Chopra P, Tinkle B, Hamonet C et al. Pain management in the Ehlers-Danlos syndromes. Am J Med Genet C Semin Med Genet 2017;175:212-9.

32 Hakim A, De Wandele I, O'Callaghan C et al. Chronic fatigue in Ehlers-Danlos syndrome-Hypermobile type. Am J Med Genet C Semin Med Genet 2017;175:175-80.

33 Fikree A, Chelimsky G, Collins H et al. Gastrointestinal involvement in the Ehlers-Danlos syndromes. Am J Med Genet C Semin Med Genet 2017:175:181-87.

34 Malhotra A, Pace A, Ruiz Maya T et al. Headaches in hypermobility syndromes: A pain in the neck? Am J Med Genet Part A 2020;182A:2902-8.

35 Grahame R. Hypermobility: an important but often neglected area within rheumatology. Nat Clin Pract Rheumatol 2008;4:522-4.

36 Tai FWD, Palsson OS, Lam CY et al. Functional gastrointestinal disorders are increased in joint hypermobility-related disorders with concomitant postural orthostatic tachycardia syndrome. Neurogastroenterol Motil 2020:e13975.

37 Hakim A, O'Callaghan C, De Wandele I et al. Cardiovascular autonomic dysfunction in Ehlers-Danlos syndrome-hypermobile type. Am J Med Genet C Semin Med Genet 2017;175:168-74.

38 Celletti C, Borsellino B, Castori $\mathrm{M}$ et al. A new insight on postural tachycardia syndrome in 102 adults with hypermobile EhlersDanlos Syndrome/hypermobility spectrum disorder. Monaldi Arch Chest Dis 2020;90.

39 Miller AJ, Stiles LE, Sheehan T et al. Prevalence of hypermobile Ehlers-Danlos syndrome in postural orthostatic tachycardia syndrome. Auton Neurosci 2020;224:102637.

40 Celletti C, Camerota F, Castori M et al. Orthostatic intolerance and postural orthostatic tachycardia syndrome in joint hypermobility syndrome/Ehlers-Danlos syndrome, hypermobility type: neurovegetative dysregulation or autonomic failure? Biomed Res Int 2017;2017:9161865.

41 Smith TO, Easton V, Bacon $\mathrm{H}$ et al. The relationship between benign joint hypermobility syndrome and psychological distress: a systematic review and meta-analysis. Rheumatology (Oxford) 2014:53:114-22.

42 Grayston R, Czanner G, Elhadd $\mathrm{K}$ et al. A systematic review and meta-analysis of the prevalence of small fiber pathology in fibromyalgia: Implications for a new paradigm in fibromyalgia etiopathogenesis. Semin Arthritis Rheum 2019;48:933-40.

43 Themistocleous AC, Ramirez JD, Serra J et al. The clinical approach to small fibre neuropathy and painful channelopathy. Pract Neurol 2014;14:368-79.

44 Sigal LH. Basic science for the clinician 53: mast cells. J Clin Rheumatol 2011:17:395-400.

45 Pardanani A. Systemic mastocytosis in adults: 2019 update on diagnosis, risk stratification and management. Am J Hematol 2019;94:363-77.

46 Seneviratne SL, Maitland A, Afrin L. Mast cell disorders in Ehlers-Danlos syndrome. Am J Med Genet C Semin Med Genet 2017:175:226-36 
47 Luzgina NG, Potapova OV, Shkurupiy VA. Structural and functional peculiarities of mast cells in undifferentiated connective tissue dysplasia. Bull Exp Biol Med 2011;150:676-8.

48 Kohn A, Chang C. The relationship between hypermobile EhlersDanlos Syndrome (hEDS), postural orthostatic tachycardia syndrome (POTS), and mast cell activation syndrome (MCAS). Clin Rev Allergy Immunol 2020;58:273-97.

49 Groven N, Fors EA, Reitan SK. Patients with fibromyalgia and chronic fatigue syndrome show increased hsCRP compared to healthy controls. Brain Behav Immun 2019;81:172-7.

50 Han CL, Sheng YC, Wang SY et al. Serum proteome profiles revealed dysregulated proteins and mechanisms associated with fibromyalgia syndrome in women. Sci Rep 2020;10:12347.

51 Eccles ], Amato M, Thompson C et al. AB0949 Autonomic and inflammatory mechanisms of pain and fatigue in fibromyalgia and ME/CFS: an interventional study. Ann Rheum Dis 2020;79:1772.

52 Seth AK, Critchley HD. Extending predictive processing to the body: emotion as interoceptive inference. Behav Brain Sci 2013;36:227-8.

53 Eccles JA, Beacher FD, Gray MA et al. Brain structure and joint hypermobility: relevance to the expression of psychiatric symptoms. Br J Psychiatry 2012;200:508-9.

54 McMorris T, Barwood M, Corbett ]. Central fatigue theory and endurance exercise: Toward an interoceptive model. Neurosci Biobehav Rev 2018;93:93-107.

55 Gonzalez Campo C, Salamone PC, Rodríguez-Arriagada N et al. Fatigue in multiple sclerosis is associated with multimodal interoceptive abnormalities. Mult Scler 2019:1352458519888881.

56 Borg C, Chouchou F, Dayot-Gorlero ] et al. Pain and emotion as predictive factors of interoception in fibromyalgia. J Pain Res 2018;11:823-35

57 Di Lernia D, Serino S, Riva G. Pain in the body. Altered interoception in chronic pain conditions: A systematic review. Neurosci Biobehav Rev 2016;71:328-41.
58 Seth AK, Suzuki K, Critchley HD. An interoceptive predictive coding model of conscious presence. Front Psychol 2011;2:395.

59 Stephan KE, Manjaly ZM, Mathys CD et al. Allostatic self-efficacy: a metacognitive theory of dyshomeostasis-induced fatigue and depression. Front Hum Neurosci 2016;10:550.

60 Eccles J. ISRCTN17018615: A novel targeted treatment for reducing anxiety in joint hypermobility. ISRCTN registry, 2020. https://doi.org/10.1186/ISRCTN17018615

61 Chan KS, Zheng JP, Mok YW et al. SARS: prognosis, outcome and sequelae. Respirology 2003;8 Suppl(Suppl 1):S36-40.

62 Carfi A, Bernabei R, Landi F et al. Persistent symptoms in patients after acute COVID-19. JAMA 2020;324:603-5.

63 National Institute for Health Research. Living with Covid19: A dynamic review of the evidence around ongoing Covid19 symptoms (often called long COVID). NIHR, 2020.

64 Esatoglu SN, Kutlubay Z, Ucar D et al. Behçet's syndrome: providing integrated care. J Multidiscip Healthc 2017:10:309-19.

65 Surrey E, Soliman AM, Trenz H et al. Impact of endometriosis diagnostic delays on healthcare resource utilization and costs. Advances in Therapy 2020;37:1087-99.

66 Youssef E, Cooper V, Delpech V et al. Barriers and facilitators to HIV testing in people age 50 and above: a systematic review. Clin Med 2017;17:508-20.

Address for correspondence: Dr Jessica A Eccles, Trafford Centre for Medical Research, University of Sussex, Falmer, Brighton BN1 9RY, UK.

Email: j.eccles@bsms.ac.uk

Twitter: @BendyBrain 